Nephron 1996;74:736-737

\title{
Acknowledgement to the Reviewers
}

\section{NEPHRON}

The editors and section editors wish to express their gratitude to the following individuals who have graciously accepted responsibility for refereeing the manuscripts which have been submitted for publication in 1996.

G. Andres, Boston, USA R. Ardaillou, Paris, France R.C. Atkins, Clayton, Australia P.-O. Attman, Göteborg, Sweden M. Aurell, Göteborg, Sweden

\section{C.A. Baldamus, Köln, Germany}

F.W. Ballardie, Manchester, Great Britain

J.E. Balow, Bethesda, USA

N. Bank, Bronx, USA

G. Barsotti, Pisa, Italy

G. Becker, Melbourne, Australia

N.H. Bell, Charleston, USA

W.M. Bennett, Portland, USA

T. Bertani, Bergamo, Italy

D.G. Bichet, Montreal, Canada

A. Bleyer, Winston Salem, USA

A. Blumberg, Aarau, Switzerland

J. Bommer, Heidelberg, Germany

G. Boner, Petah Tiqvah, Israel

W. Border, Salt Lake City, USA

M. Broyer, Paris, France

G. Camussi, Torino, Italy

A. Canepa, Genova, Italy

G. Capasso, Napoli, Italy

G.R.D. Catto, Aberdeen, United Kingdom T.M. Chan, Pokfulam, Hongkong

B. Charra, Tassin, France

A.K. Cheung, Salt Lake City, USA J. Churg, Paterson, USA F.L. Coe, Chicago, USA G. Coen, Roma, Italy A. Cupisti, Pisa, Italy

W.H. Dantzler, Tucson, USA

A.M. Davison, Leeds, United Kingdom

M.E. De Broe, Edegem-Antwerp, Belgium

N.G De Santo, Napoli, Italy

V. Delaney, Valhalla, USA

B.G. Delano, Brooklyn, USA

CJ. Diskin, Opelika, USA

AJ.M. Donker, Amsterdam, 
The Netherlands D. Droz, Paris, France T.B. Drüeke, Paris, France A. Dvilansky, Beer Sheva, Israel L.D. Dworkin, Providence, USA

CM. Edelmann, Bronx, USA

G. Eknoyan, Houston, USA

H.E. Eliahou, Ramat-Gan, Israel

B.T. Emmerson, Woolloongabba, Australia

F.E. Epstein, Boston, USA

D.B. Evans, Cambridge, Great Britain

K.F. Fairley, Richmond, Australia

RJ. Falk, Chapel Hill, USA

P. Fauchald, Blommenholm, Norway

J.W. Fisher, New Orleans, USA

J. Floege, Seattle, USA

M.M. Friedlaender, Jerusalem, Israel

EA. Friedman, Brooklyn, USA

M.H. Gault, St. John's, Canada B.G. Gauthier, New Hyde Park, USA

B. Goldberg, Birnan Johannesburg,

South Africa P. Goldwasser, Brooklyn, USA L. Gotloib, Afula, Israel R. Greger, Freiburg i.Br., Germany P. Gross, Berlin, Germany J.P. Grünfeld, Paris, France

R.M. Hakim, Nashville, USA

J.S. Han, Seoul, South Corea

N.K. Hollenberg, Boston, USA

W.H. Hörl, Vienna, Austria

LB. Houston, Manchester, Great Britain

L.S. Ibels, St. Leonards, Australia I. Ichikawa, Nashville, USA K. Iseki, Okinawa, Japan I.

Ishikawa, Ishikawa, Japan

C. Jacobs, Paris, France

J.C. Jennette, Chapel Hill, USA

M.J. Karnovsky, Boston, USA M. Kashgarian, New Haven, USA W.F. Keane, Minneapolis, USA D.N.S. Kerr, London, Great Britain P. Keshaviah, Minneapolis, USA P.L. Kimmel, Washington, USA CM. Kjellstrand, Edmonton, Canada C.R. Kleeman, Los Angeles, USA J.P. Knochel, Dallas, USA K.-M. Koch, Hannover, Germany H. Koide, Tokyo, Japan F. Kokot, Katowice, Poland CM. Kunin, Columbus, USA

F. Lang, Tubingen, Germany H.A. Lee, Portsmouth,

United Kingdom M. Levi, Dallas, USA

C.-Y. Lin, Taipei, Taiwan

F. Llach, Newark, USA

D. McCarron, Portland, USA

G.A. MacGregor, London, Great Britain

K. Maeda, Nagoya, Japan

N.P. Mallick, Manchester, Great Britain

A.K. Mandal, Dayton, USA

M. Martinez-Maldonado, Atlanta, USA

G. Maschio, Verona, Italy

A.P. Maxwell, Belfast, United Kingdom K.-H. Meyer zum Büschenfelde, 
Mainz, Germany A. Meyrier, Bobigny, France A.F. Michael, Minneapolis, USA KA. Miles, Brisbane, Australia H. Mori, Osaka, Japan R.A. Mustard, Toronto, Canada

H. Nakahama, Hyogo, Japan J. Neugarten, Bronx, USA S. Nilwarangkur, Chimplee,

Talingchun, Bangkok, Thailand R. Nitschke, Freiburg i.Br., Germany E.P. Nord, Stony Brook, USA B.E.C Nordin, Adelaide, Australia

M.S. Oh, Brooklyn, USA

T. Okasora, Yonago, Japan

D.G. Oreopoulos, Toronto, Canada

CE. Palant, Reno, USA A. Pasternack, Tampere, Finland A.J. Pesce, Cincinnati, USA T.M.

Phillips, Washington, USA J. Platt, Durham, USA V.E. Pollak, Cincinnati, USA C Ponticelli, Milano, Italy G.A. Porter, Portland, USA J.G Porush, Brooklyn, USA H.G Preuss, Washington, USA M. Puoti, Brescia, Italy

E. Quellhorst, Hannover-Münden,

Germany

T.K.S. Rao, Brooklyn, USA G. Remuzzi, Bergamo, Italy J.P. Revillard, Lyon, France M.J.

Richard, Grenoble, France E. Ritz, Heidelberg, Germany A. Rofe, Adelaide, Australia D. Roth, Miami, USA

736

S. Sabatini, Lubbock, USA

A. Sacripante, Pisa, Italy

A. Salvetti, Pisa, Italy

F.P. Schena, Bari, Italy

V. Schettler, Göttingen, Germany

R.W. Schrier, Denver, USA

G. Schulman, Nashville, USA

R. Shainkin-Kestenbaum, Kfar Saba, Israel

S. Shaldon, Montpellier, France

R.A. Sherman, New Brunswick, USA

D.A. Sica, Richmond, USA

H.G. Sieberth, Aachen, Germany

J.-D. Sraer, Paris, France

J. Strauss, Miami, USA

G.E. Striker, Bethesda, USA

L.J. Striker, Bethesda, USA

D.E.R. Sutherland, Minneapolis, USA

P.I. Terasaki, Los Angeles, USA K. Thomsen, Risskov, Denmark N.M. Thomson, Prahran, Melbourne,

Australia Y. Tomino, Tokyo, Japan

N. Ueda, Little Rock, USA

D.E. Uehlinger, Bern, Switzerland

C.A. Vaamonde, Miami, USA

N.D. Vaziri, Orange, USA

G.C. Viberti, London, United Kingdom

J. Walls, Leicester, Great Britain E.N. Wardle, North Leigh, Oxford, United Kingdom L.G. Wesson, Lansdale, USA CD. West, Cincinnati, USA

C. van Ypersele, Bruxelles, Belgium 
E.T. Zawada, Sioux Falls, USA P. Zucchelli, Bologna, Italy

Acknowledgement to the Reviewers

73' 